PROCEEDINGS OF THE

AMERICAN MATHEMATICAL SOCIETY

Volume 128, Number 12, Pages 3579-3586

S 0002-9939(00)05886-X

Article electronically published on August 17, 2000

\title{
COMPOSITION OPERATORS ON DIRICHLET-TYPE SPACES
}

\author{
R. A. HIBSCHWEILER
}

(Communicated by David R. Larson)

\begin{abstract}
The Dirichlet-type space $D^{p}(1 \leq p \leq 2)$ is the Banach space of functions analytic in the unit disc with derivatives belonging to the Bergman space $A^{p}$. Let $\Phi$ be an analytic self-map of the disc and define $C_{\Phi}(f)=$ $f \circ \Phi$ for $f \in D^{p}$. The operator $C_{\Phi}: D^{p} \rightarrow D^{p}$ is bounded (respectively, compact) if and only if a related measure $\mu_{p}$ is Carleson (respectively, compact Carleson). If $C_{\Phi}$ is bounded (or compact) on $D^{p}$, then the same behavior holds on $D^{q}(1 \leq q<p)$ and on the weighted Dirichlet space $D_{2-p}$. Compactness on $D^{p}$ implies that $C_{\Phi}$ is compact on the Hardy spaces and the angular derivative exists nowhere on the unit circle. Conditions are given which, together with the angular derivative condition, imply compactness on the space $D^{p}$. Inner functions which induce bounded composition operators on $D^{p}$ are discussed briefly.
\end{abstract}

\section{INTRODUCTION}

Let $D=\{z:|z|<1\}$ and let $A$ denote normalized area measure on $D$. The Bergman space $A^{p}(1 \leq p<\infty)$ is the Banach space of functions $f$ analytic in the $\operatorname{disc} D$ such that $\|f\|_{A^{p}}^{p}=\int_{D}|f(z)|^{p} d A(z)<\infty$. The Dirichlet-type space $D^{p}$ is the set of functions analytic in $D$ with derivatives belonging to $A^{p}$. The set $D^{p}$ is a Banach space, with norm given by

$$
\|f\|_{D^{p}}=|f(0)|+\left\|f^{\prime}\right\|_{A^{p}} .
$$

Note that point evaluation is continuous on $D^{p}$ and $D^{p} \subset D^{q}$ if $1 \leq q<p$.

Throughout this paper, $\Phi$ denotes an analytic self-map of $D$. The composition operator $C_{\Phi}$ is defined by $C_{\Phi}(f)=f \circ \Phi$ for $f \in D^{p}$. If $C_{\Phi}(f) \in D^{p}$ for every $f \in D^{p}$, then $C_{\Phi}$ is bounded, by the Closed Graph Theorem.

Interest in the spaces $D^{p}$ is motivated by the work of R. Roan [19] and B. D. MacCluer 14, who studied composition operators on $S^{p}$, the space of functions with derivatives in the Hardy space $H^{p}$ for $p \geq 1$. Other related work appears in 15, where MacCluer and J. H. Shapiro studied composition operators on the weighted Dirichlet spaces $D_{\alpha}$.

\section{SECTION 1}

Let $\mu$ be a finite positive Borel measure on $D$. For $|\zeta|=1$ and $0<\delta \leq 2$, $S(\zeta, \delta)$ is the Carleson set $\{z \in D:|z-\zeta|<\delta\}$. The measure $\mu$ is said to be

Received by the editors October 16, 1998 and, in revised form, February 12, 1999.

2000 Mathematics Subject Classification. Primary 47B38; Secondary 30H05.

Key words and phrases. Composition operator, Dirichlet space, Carleson measure, angular derivative. 
Carleson if there is a constant $C$ such that $\mu(S(\zeta, \delta)) \leq C \delta^{2}$ for all $\zeta$ and $\delta$. The measure is said to be compact Carleson if

$$
\lim _{\delta \rightarrow 0} \sup _{|\zeta|=1} \frac{\mu(S(\zeta, \delta))}{\delta^{2}}=0
$$

Carleson measures have been useful in the study of composition operators in several settings [11, 13, 14, 15, 27, 28].

For $w \in D$, let $N_{2}(\Phi, w)$ denote the number of zeroes (counting multiplicities) of the equation $\Phi(z)-w$. For $1 \leq p<2$ and $w \in D, N_{p}(\Phi, w)$ is defined to be the modified counting function

$$
N_{p}(\Phi, w)=\sum \frac{1}{\left|\Phi^{\prime}(z)\right|^{2-p}}
$$

where the sum extends over the zeroes of $\Phi-w$, repeated by multiplicity. In particular, $N_{p}(\Phi, w)=0$ for $w \notin \Phi(D)$. Let $\mu_{p}$ be the measure defined on $D$ by $d \mu_{p}(w)=N_{p}(\Phi, w) d A(w), 1 \leq p \leq 2$. Note that $\mu_{p}$ is a finite measure if and only if $\Phi \in D^{p}$.

The proofs of the first two theorems are standard. A sketch of the proofs is provided in Section 3.

Theorem 1. The operator $C_{\Phi}$ is bounded on $D^{p}$ if and only if $\mu_{p}$ is a Carleson measure.

Theorem 2. The operator $C_{\Phi}$ is compact on $D^{p}$ if and only if the measure $\mu_{p}$ is compact Carleson.

The case $p=2$ in Theorems 1 and 2 was first proven by MacCluer and Shapiro in their study of the weighted Dirichlet spaces $D_{\alpha}$ 15. An analytic function $f$ belongs to $D_{\alpha}(\alpha>-1)$ if $\int_{D}\left|f^{\prime}(z)\right|^{2}\left(1-|z|^{2}\right)^{\alpha} d A(z)<\infty$. Note that $D_{0}=D^{2}$, the classical Dirichlet space. For $p=2$ the norm (1) is equivalent to the norm used in [15] for $D_{0}$.

Throughout the remainder of this work $C$ will denote a positive constant, the exact value of which will vary from one appearance to the next.

Theorem 3. If $C_{\Phi}$ is bounded (respectively, compact) on $D^{p}$ and $1 \leq q<p$, then $C_{\Phi}$ is bounded (respectively, compact) on $D^{q}$.

Proof. The hypotheses imply that $\Phi \in D^{q}$ for $1 \leq q \leq p$. Thus $\mu_{q}$ is a finite measure on the disc. Since $q<p$, Hölder's Inequality implies that

$$
\begin{gathered}
\mu_{q}(S(\zeta, \delta))=\int_{\Phi^{-1}(S(\zeta, \delta))}\left|\Phi^{\prime}\right|^{q} d A \\
\leq\left(\int_{\Phi^{-1}(S(\zeta, \delta))}\left|\Phi^{\prime}\right|^{p} d A\right)^{q / p}\left(\int_{\Phi^{-1}(S(\zeta, \delta))} 1 d A\right)^{(p-q) / p} \\
=\mu_{p}(S(\zeta, \delta))^{q / p} A \Phi^{-1}(S(\zeta, \delta))^{(p-q) / p} .
\end{gathered}
$$$$
\text { Since } C_{\Phi} \text { is bounded on the Bergman spaces, Theorem } 4.3 \text { [15] implies that the }
$$
measure $A \Phi^{-1}$ is Carleson. Thus (2) yields

$$
\mu_{q}(S(\zeta, \delta)) \leq C \delta^{2(p-q) / p} \mu_{p}(S(\zeta, \delta))^{q / p} .
$$

If $C_{\Phi}$ is bounded on $D^{p}$, Theorem 1 implies that there is a constant $C$ such that $\mu_{p}(S(\zeta, \delta)) \leq C \delta^{2}$ for all $\zeta$ and $\delta$ as described above. Thus (3) shows that $\mu_{q}(S(\zeta, \delta)) \leq C \delta^{2}$. By a second application of Theorem $1, C_{\Phi}$ is bounded on $D^{q}$. 
If $C_{\Phi}$ is compact on $D^{p}$, then $\mu_{p}(S(\zeta, \delta)) / \delta^{2} \rightarrow 0$ uniformly on $\{\zeta:|\zeta|=1\}$ as $\delta \rightarrow 0$. Relation (3) shows that $\mu_{q}$ is a compact Carleson measure. By Theorem 2, $C_{\Phi}$ is compact on $D^{q}$.

The following corollary is an immediate consequence of Theorems 2 and 3.

Corollary 1. 1. If $\Phi$ is an analytic self-map of $D$ with bounded multiplicity, then $C_{\Phi}$ is bounded on $D^{p}$ for $1 \leq p \leq 2$.

2. If $\Phi \in D^{p}$ and $\|\Phi\|_{\infty}<1$, then $C_{\Phi}$ is compact on $D^{q}$ for $1 \leq q \leq p$.

In [15, p. 892] MacCluer and Shapiro defined a measure $\nu_{\alpha}$ on the disc by $d \nu_{\alpha}(z)=\left|\Phi^{\prime}(z)\right|^{2}\left(1-|z|^{2}\right)^{\alpha} d A(z)(\alpha>-1)$. The operator $C_{\Phi}$ is bounded (respectively, compact) on the weighted Dirichlet space $D_{\alpha}$ if and only if the measure $\nu_{\alpha} \Phi^{-1}$ is $\alpha$-Carleson (respectively, compact $\alpha$-Carleson). Details can be found in [15].

The space $D_{\alpha}$ is said to be heavily weighted if $-1<\alpha<0$. If $C_{\Phi}$ is bounded (respectively, compact) on a heavily weighted space, then $C_{\Phi}$ is bounded (compact) on $D_{0}=D^{2}$ 15. Theorem 3 now implies that $C_{\Phi}$ is bounded (compact) on $D^{p}$ for $1 \leq p<2$. Shapiro has shown that compactness on $D_{\alpha}$ for $-1<\alpha<0$ is equivalent to the conditions $\Phi \in D_{\alpha}$ and $\|\Phi\|_{\infty}<1[21]$.

Theorem 4. For $1 \leq p \leq 2$, let $\alpha=2-p$. If $C_{\Phi}$ is bounded (respectively, compact) on $D^{p}$, then $C_{\Phi}$ is bounded (compact) on the weighted Dirichlet space $D_{\alpha}$.

Proof. It is enough to consider $1 \leq p<2$.

Suppose that $C_{\Phi}$ is bounded on $D^{p}$ and let $\alpha=2-p$. Then

$$
\nu_{\alpha}(D)=\int_{D}\left|\Phi^{\prime}(z)\right|^{p}\left|\Phi^{\prime}(z)\right|^{\alpha}\left(1-|z|^{2}\right)^{\alpha} d A(z) .
$$

Since $\Phi$ is an analytic self-map of the unit disc, the Schwarz-Pick Lemma guarantees that

$$
\frac{1-|z|^{2}}{1-|\Phi(z)|^{2}}\left|\Phi^{\prime}(z)\right| \leq 1
$$

Thus (4) implies that

$$
\nu_{\alpha}(D) \leq \int_{D}\left|\Phi^{\prime}(z)\right|^{p}\left(1-|\Phi(z)|^{2}\right)^{\alpha} d A(z) \leq \int_{D}\left|\Phi^{\prime}\right|^{p} d A .
$$

Since $\Phi \in D^{p}$, it follows that $\nu_{\alpha}$ is a finite measure on $D$.

It remains to prove that $\nu_{\alpha} \Phi^{-1}(S(\zeta, \delta)) \leq C \delta^{2+\alpha}$ for all $|\zeta|=1$ and $0<\delta \leq 2$. By the argument given in the previous paragraph,

$$
\nu_{\alpha} \Phi^{-1}(S(\zeta, \delta)) \leq \int_{\Phi^{-1}(S(\zeta, \delta))}\left|\Phi^{\prime}(z)\right|^{p}\left(1-|\Phi(z)|^{2}\right)^{\alpha} d A(z) .
$$

For $z \in \Phi^{-1}(S(\zeta, \delta)), 1-|\Phi(z)|<\delta$. Thus

$$
\nu_{\alpha} \Phi^{-1}(S(\zeta, \delta)) \leq(2 \delta)^{\alpha} \int_{\Phi^{-1}(S(\zeta, \delta))}\left|\Phi^{\prime}\right|^{p} d A=(2 \delta)^{\alpha} \mu_{p}(S(\zeta, \delta)) .
$$

Since $C_{\Phi}$ is bounded on $D^{p}$, Theorem 1 implies that $\mu_{p}(S(\zeta, \delta))<C \delta^{2}$. It follows that the measure $\nu_{\alpha} \Phi^{-1}$ is $\alpha$-Carleson, as required.

The assertion about compactness is proved in a similar way. 
The following theorem was first suggested by Shapiro [23].

Theorem 5. If $C_{\Phi}$ is compact on $D^{p}$ for some $1 \leq p \leq 2$, then $C_{\Phi}$ is compact on the Hardy spaces.

Proof. Since $C_{\Phi}$ is compact on $D^{p}$, Theorem 3 implies that $C_{\Phi}$ is compact on $D^{1}$. Theorem 4 now implies that $C_{\Phi}$ is compact on the weighted Dirichlet space $D_{1}=H^{2}$. As noted in [25], compactness on $H^{2}$ is equivalent to compactness on $H^{q}$ for $0<q<\infty$.

Corollary 2. 1. If $\Phi$ is an inner function, then $C_{\Phi}$ is not compact on $D^{p}$ for any $1 \leq p \leq 2$.

2. If $C_{\Phi}$ is compact on $D^{p}$, then the angular derivative exists nowhere on the circle.

3. If $C_{\Phi}$ is compact on $D^{p}$, then $C_{\Phi}$ is compact on $A_{\alpha}^{q}$ for $0<q<\infty$ and $\alpha>-1$.

4. Suppose that $\Phi^{\prime}$ is bounded. The operator $C_{\Phi}$ is compact on $D^{p}$ for all $1 \leq$ $p \leq 2$ if and only if $\|\Phi\|_{\infty}<1$.

Proof. The first three assertions follow from Theorem 5 and the well-known results about compactness on the Hardy spaces and the weighted Bergman spaces [15].

Suppose that $\Phi^{\prime}$ is bounded and $\|\Phi\|_{\infty}=1$. It follows that the angular derivative is finite at some point on the circle, and thus $C_{\Phi}$ is not compact on $D^{p}$.

The remaining implication follows from Corollary 1.

There exist inner functions $\Phi$ with no finite angular derivative at any point on the circle. Thus the converse of the second assertion in the corollary is false.

Shapiro [21] has shown that there are functions $\Phi$ with $\|\Phi\|_{\infty}=1$ such that $C_{\Phi}$ is compact on $D^{2}$, the Dirichlet space. Theorem 3 implies that $C_{\Phi}$ is compact on $D^{p}$ for $1 \leq p \leq 2$. Thus the condition $\|\Phi\|_{\infty}<1$ is not necessary for $C_{\Phi}$ to be compact on the Dirichlet-type spaces $D^{p}$. In comparison, MacCluer proved that $C_{\Phi}$ is compact on $S^{p}(1 \leq p<\infty)$ if and only if $\Phi \in S^{p}$ and $\|\Phi\|_{\infty}<1$ [14].

Theorem 6. Suppose $\|\Phi\|_{\infty}=1$. If $C_{\Phi}$ is bounded on $D^{p}$ and the angular derivative of $\Phi$ exists at no point of the circle, then $C_{\Phi}$ is compact on $D^{q}$ for $1 \leq q<p$.

Proof. As in the proof of Theorem 3, the hypotheses imply that $\mu_{q}$ is a finite measure and

$$
\frac{\mu_{q}(S(\zeta, \delta))}{\delta^{2}} \leq\left(\frac{\mu_{p}(S(\zeta, \delta))}{\delta^{2}}\right)^{q / p}\left(\frac{A \Phi^{-1}(S(\zeta, \delta))}{\delta^{2}}\right)^{(p-q) / p} .
$$

Since the angular derivative of $\Phi$ exists nowhere on the unit circle, the measure $A \Phi^{-1}$ is compact Carleson [15]. Thus the second expression on the right in (5) tends to 0 uniformly on $\{\zeta:|\zeta|=1\}$ as $\delta \rightarrow 0$. Because $C_{\Phi}$ is bounded on $D^{p}$, the first expression on the right is bounded. Thus the measure $\mu_{q}$ is compact Carleson, and Theorem 2 implies the result.

If $\Phi$ is bounded multiplicity, then $C_{\Phi}$ is compact on the Hardy spaces if and only if the angular derivative of $\Phi$ exists nowhere on the circle [15]. Theorem 7 states the analogous result for the $D^{p}$ spaces, $1 \leq p<2$. 
Theorem 7. Suppose that $\Phi$ is of bounded multiplicity and \|\|$_{\infty}=1$. The following are equivalent:

1. The operator $C_{\Phi}$ is compact on $D^{p}$ for all $p, 1 \leq p<2$.

2. $C_{\Phi}$ is compact on the Hardy spaces.

3. The angular derivative of $\Phi$ exists at no point on the circle.

Proof. It suffices to prove that the third assertion implies the first.

Since $\Phi$ has bounded multiplicity, $C_{\Phi}$ is bounded on $D^{2}$. Theorem 6 now implies the result.

In [11], Jovovic and MacCluer point out that a univalent function mapping $D$ onto a non-tangential approach region will not induce a compact composition operator on the Dirichlet space. Thus univalence and non-existence of the angular derivative are not sufficient to imply that $C_{\Phi}$ is compact on $D^{2}$.

\section{SECTION 2}

In this section we present a brief discussion of inner functions which induce bounded composition operators on the space $D^{p}$.

If $\Phi$ is a finite Blaschke product, then $\Phi^{\prime}$ is bounded, and thus $\Phi$ induces a composition operator on $D^{p}$ for $1 \leq p \leq 2$. D. J. Newman and H. S. Shapiro proved that if $\Phi$ is inner and $\Phi \in D^{2}$, then $\Phi$ is a finite Blaschke product [16]. If $\Phi$ is an infinite Blaschke product or a singular inner function, then $\Phi$ need not belong to $D^{1}$ 3. 20]. Since $D^{p} \subset D^{1}$ for $1 \leq p \leq 2$, this shows that such an inner function may fail to induce a bounded composition operator on any of the Dirichlet-type spaces.

As a positive example, let $\Lambda(z)=e^{-(1+z) /(1-z)}$ for $z \in D$. It is clear that $\Lambda \notin D^{2}$. Let $w \in \Lambda(D)=D \backslash\{0\}$ and denote the preimages of $w$ by $z_{n}(w), n \in \mathbf{Z}$. For $1 \leq p<2$,

$$
N_{p}(\Lambda, w)=\sum_{n \in \mathbf{Z}} \frac{\left|1-z_{n}(w)\right|^{2(2-p)}}{(2|w|)^{2-p}} .
$$

There is a natural number $N$ and a positive constant $C_{1}$ independent of $w$ such that $C_{1}<|n|\left|1-z_{n}(w)\right|$ for $|w|>1 / 2$ and $|n|>N$. It follows that $N_{3 / 2}(\Lambda, w)=\infty$ for all $|w|>1 / 2$, and thus $\Lambda \notin D^{p}$ for $3 / 2 \leq p \leq 2$.

By similar reasoning there is a positive constant $C_{2}$ and a natural number $N$ such that $|n|\left|1-z_{n}(w)\right|<C_{2}$ for all $|n|>N$ and all $w \in \Lambda(D)$. It follows that $N_{p}(\Lambda, w)<C|w|^{p-2}$ for $1 \leq p<3 / 2$. Thus $\mu_{p}$ is a finite measure. A calculation shows that $\mu_{p}$ is Carleson. By Theorem $1 C_{\Lambda}$ is bounded on $D^{p}$ if and only if $1 \leq p<3 / 2$.

As a generalization of this example let $F$ be defined by

$$
F(z)=\int_{\{x:|x|=1\}} \frac{1+x z}{1-x z} d \mu(x)
$$

where $\mu$ is a convex combination of finitely many point masses on the circle. For $z \in D$, define $\Psi(z)=e^{-F(z)}$ and $h(z)=(F(z)-1) /(F(z)+1)$. Then $h$ is an analytic self-map of the disc and $\Psi=\Lambda \circ h$. Since $h$ is rational and $\left|h\left(e^{i \theta}\right)\right|=1$ for $0 \leq \theta<2 \pi, h$ is a finite Blaschke product. It follows that $C_{\Psi}$ is a bounded operator on $D^{p}$ for $1 \leq p<3 / 2$. 
We close this section by noting a connection between the Besov spaces $B_{\gamma}$ and the spaces $D^{p}$. A function $f$ analytic in $D$ belongs to $B_{\gamma}(0<\gamma<1)$ if

$$
\int_{0}^{2 \pi} \int_{0}^{1}\left|f\left(r e^{i \theta}\right)\right|(1-r)^{1 / \gamma-2} d r d \theta<\infty .
$$

A result of Hardy and Littlewood [9, p. 84] implies that $B_{\gamma} \subset A^{2 \gamma}$. Several authors have studied conditions on inner functions $\Phi$ sufficient to imply that $\Phi^{\prime} \in B_{\gamma}$, and thus, $\Phi \in D^{2 \gamma}[1,2,13,8,18,20$.

\section{SECTION 3}

The proofs of Theorems 1 and 2 will be presented in this section. The proofs depend upon Theorem 4.3 in [15], which states that for a finite positive measure $\nu$, the identity map $I: A^{p} \rightarrow L^{p}(\nu)$ is bounded (respectively, compact) if and only if $\nu$ is Carleson (respectively, compact Carleson).

We may assume that $\Phi$ is not constant. Unless otherwise indicated, all integrals in this section are taken over the unit disc $D$.

For the proof of Theorem 1, assume that $C_{\Phi}$ is bounded on $D^{p}$. Let $f \in D^{p}$ with $f(0)=0$. The hypothesis implies that

$$
\int\left|f^{\prime} \circ \Phi\right|^{p}\left|\Phi^{\prime}\right|^{p} d A \leq C \int\left|f^{\prime}\right|^{p} d A
$$

By a change of variable (see [7], p. 36) and the definition of $\mu_{p}$,

$$
\int\left|f^{\prime} \circ \Phi\right|^{p}\left|\Phi^{\prime}\right|^{p} d A=\int\left|f^{\prime}\right|^{p} d \mu_{p}
$$

Let $g \in A^{p}$ and define $f(z)=\int_{0}^{z} g(w) d w$. The argument above shows that

$$
\int|g|^{p} d \mu_{p} \leq C \int|g|^{p} d A
$$

and it follows that $\mu_{p}$ is a Carleson measure.

For the converse, suppose that $\mu_{p}$ is a Carleson measure. Then (7) holds for every $g \in A^{p}$. It follows that

$$
\int\left|(f \circ \Phi)^{\prime}\right|^{p} d A=\int\left|f^{\prime}\right|^{p} d \mu_{p} \leq C \int\left|f^{\prime}\right|^{p} d A \leq C\|f\|_{D^{p}}^{p}
$$

for every $f \in D^{p}$. Since evaluation at $\Phi(0)$ is a bounded linear functional on $D^{p}$, the argument shows that $C_{\Phi}$ is a bounded operator on $D^{p}$. The proof of Theorem 1 is complete.

For the proof of Theorem 2, note that $C_{\Phi}: D^{p} \rightarrow D^{p}$ is compact if and only if $\left\|f_{n} \circ \Phi\right\|_{D^{p}} \rightarrow 0$ for any bounded sequence $\left(f_{n}\right)$ in $D^{p}$ with $f_{n} \rightarrow 0$ uniformly on compact subsets.

Suppose that $C_{\Phi}$ is compact on $D^{p}$. Let $\left(g_{n}\right)$ be a bounded sequence in $A^{p}$ with $g_{n} \rightarrow 0$ uniformly on compact subsets, and define $f_{n}(z)=\int_{0}^{z} g_{n}(w) d w$. The hypothesis implies $\left\|g_{n}\right\|_{L^{p}\left(\mu_{p}\right)}=\left\|\left(f_{n} \circ \Phi\right)^{\prime}\right\|_{A^{p}} \rightarrow 0$ as $n \rightarrow \infty$ and thus $\mu_{p}$ is compact Carleson.

For the converse, assume that $\mu_{p}$ is compact Carleson. Suppose $\left\|f_{n}\right\|_{D^{p}} \leq 1$ and $f_{n} \rightarrow 0$ uniformly on compact subsets. Since $I: A^{p} \rightarrow L^{p}\left(\mu_{p}\right)$ is compact, it follows that $\left\|\left(f_{n} \circ \Phi\right)^{\prime}\right\|_{A^{p}}=\left\|f_{n}^{\prime}\right\|_{L^{p}\left(\mu_{p}\right)} \rightarrow 0$. Since $\left|f_{n}(\Phi(0))\right| \rightarrow 0$, the argument yields $\left\|f_{n} \circ \Phi\right\|_{D^{p}} \rightarrow 0$. Thus $C_{\Phi}$ is compact on $D^{p}$. 


\section{ACKNOWLEDGEMENT}

The author would like to thank J. H. Shapiro for many helpful conversations.

\section{REFERENCES}

[1] P. R. Ahern and D. N. Clark, On inner functions with $B^{p}$ derivative, Mich. Math. J. 23 (1976), 107-118. MR 54:2976

[2] P. R. Ahern and D. N. Clark, On inner functions with $H^{p}$ derivative, Mich. Math. J. 21 (1974), 115-127. MR 49:9218

[3] H. A. Allen and C. L. Belna, Singular inner functions with derivative in $B^{p}$, Mich. Math. J. 19 (1972), 185-188. MR 45:8844

[4] K. R. M. Attele, Analytic multipliers of Bergman spaces, Mich. Math. J. 31 (1984), 307-319. MR 86g:46039

[5] S. Axler, Multiplication operators on Bergman spaces, J. Reine Angewandt Math. 336 (1982), 26-44. MR 84b:30052

[6] J. A. Cima and W. R. Wogen, A Carleson measure theorem for the Bergman space on the ball, J. Operator Theory 7 (1982), 157-165. MR 83f:46022

[7] C. C. Cowen and B. D. MacCluer, Composition Operators on Spaces of Analytic Functions, CRC Press, Boca Raton, 1995. MR 97i:47056

[8] M. R. Cullen, Derivatives of singular inner functions, Mich. Math. J. 18 (1971), 283-287. MR 44:438

[9] P. Duren, Theory of $H^{p}$ Spaces, Academic Press, New York, 1970. MR 42:3552

[10] W. Hastings, A Carleson measure theorem for Bergman spaces, Proc. Amer. Math. Soc. 52 (1975), 237-241. MR 51:11082

[11] M. Jovovic and B. D. MacCluer, Composition operators on Dirichlet spaces, Acta Sci. Math. (Szeged) 63 (1997), 229-247. MR 98d:47067

[12] R. Kerman and E. Sawyer, Carleson measures and multipliers of Dirichlet-type spaces, Trans. Amer. Math. Soc. 309 (1988), 87-98. MR 89i:30044

[13] B. D. MacCluer, Compact composition operators on $H^{p}\left(B_{N}\right)$, Mich. Math. J. 32 (1985), 237-248. MR 86g:47037

[14] B. D. MacCluer, Composition operators on $S^{p}$, Houston J. Math. 13 (1987), 245-254. MR 88h:47044

[15] B. D. MacCluer and J. H. Shapiro, Angular derivatives and compact composition operators on the Hardy and Bergman spaces, Can. J. Math., Vol. 38 (1986), 878-906. MR 87h:47048

[16] D. J. Newman and H. S. Shapiro, The Taylor coefficients of inner functions, Mich. Math. J. 9 (1962), 249-255. MR 26:6371

[17] G. Piranian, Bounded functions with large circular variation, Proc. Amer. Math. Soc. 19 (1968), 1255-1257. MR 37:6464

[18] D. Protas, Blaschke products with derivative in $H^{p}$ and $B^{p}$, Mich. Math. J. 20 (1973), 393396. MR 49:9217

[19] R. Roan, Composition operators on the space of functions with $H^{p}$-derivative, Houston J. Math. 4 (1978), 423-438. MR 58:23735

[20] W. Rudin, The radial variation of analytic functions, Duke Math. J. 22 (1955), 235-242. MR 18:27g

[21] J. H. Shapiro, Compact composition operators on spaces of boundary-regular holomorphic functions, Proc. Amer. Math. Soc. 100 (1987), 49-57. MR 88c:47059

[22] J. H. Shapiro, Composition Operators and Classical Function Theory, Springer-Verlag, New York, 1993. MR 94k:47049

[23] J. H. Shapiro, private communication.

[24] J. H. Shapiro, The essential norm of a composition operator, Annals of Math. 125 (1987), 375-404. MR 88c:47058

[25] J. H. Shapiro and P. D. Taylor, Compact, nuclear, and Hilbert-Schmidt composition operators on $H^{2}$, Indiana Univ. Math. J. 23 (1973), 471-496. [MR 48:4816]

[26] D. A. Stegenga, Multipliers of the Dirichlet space, Illinois J. Math 24 (1980), 113-139. MR 81a:30027 
[27] N. Zorboska, Composition operators on $S_{a}$ spaces, Indiana University Math. J. 39 (1990), 847-857. MR 91k:47070

[28] N. Zorboska, Composition operators on weighted Dirichlet spaces, Proc. Amer. Math. Soc. 126 (1998), 2013-2023. MR 98h:47047

Department of Mathematics, University of New Hampshire, Durham, New Hampshire 03824

E-mail address: rah2@cisunix.unh.edu 